\title{
Rapid detection of multidrug-resistant Mycobacterium tuberculosis using the malachite green decolourisation assay
}

\author{
Ahmet Yilmaz Coban ${ }^{1 /+}$, Meltem Uzun² \\ ${ }^{1}$ Department of Medical Microbiology, Medical School, Ondokuz Mayis University, Samsun, Turkey \\ ${ }^{2}$ Department of Medical Microbiology, Istanbul Medical School, Istanbul University, Istanbul, Turkey
}

\begin{abstract}
Early detection of drug resistance in Mycobacterium tuberculosis isolates allows for earlier and more effective treatment of patients. The aim of this study was to investigate the performance of the malachite green decolourisation assay (MGDA) in detecting isoniazid (INH) and rifampicin (RIF) resistance in $\mathrm{M}$. tuberculosis clinical isolates. Fifty M. tuberculosis isolates, including 19 multidrug-resistant, eight INH-resistant and 23 INH and RIF-susceptible samples, were tested. The sensitivity, specificity, positive predictive value (PPV), negative predictive value (NPV) and agreement of the assay for INH were $92.5 \%, 91.3 \%, 92.5 \%, 91.3 \%$ and $92 \%$, respectively. Similarly, the sensitivity, specificity, PPV, NPV and agreement of the assay for RIF were $94.7 \%, 100 \%, 100 \%, 96.8 \%$ and $98 \%$, respectively. There was a major discrepancy in the tests of two isolates, as they were sensitive to INH by the MGDA test, but resistant by the reference method. There was a minor discrepancy in the tests of two additional isolates, as they were sensitive to INH by the reference method, but resistant by the MGDA test. The drug susceptibility test results were obtained within eight-nine days. In conclusion, the MGDA test is a reliable and accurate method for the rapid detection of INH and RIF resistance compared with the reference method and the MGDA test additionally requires less time to obtain results.
\end{abstract}

Key words: Mycobacterium tuberculosis - malachite green decolourisation assay - susceptibility testing - isoniazid - rifampicin

Early detection of drug resistance in Mycobacterium tuberculosis isolates allows for appropriate and timely treatment (Martin et al. 2008). There are many welldefined phenotypic methods for testing the drug susceptibility of $M$. tuberculosis. The proportion method, performed on Löwenstein-Jensen and 7H10 or 7H11 agar media, is recommended as a reference method. However, this method requires at least three-six weeks to obtain results (Kent \& Kubica 1985). There are also rapid automated systems for drug susceptibility testing. The BACTEC 460TB system (Becton Dickinson Diagnostic Systems, Sparks, MD, USA), which is no longer commercially available, is a semi-automated rapid system that is expensive and contains radioactive material. The BACTEC MGIT 960 system (Becton Dickinson Diagnostic Systems) is a non-radiometric rapid automated system. Finally, molecular methods of susceptibility testing are available, including the expensive commercial Xpert MTB/RIF and Genotype MTBDRplus assays (Bwanga et al. 2009, Friedrich et al. 2011, Chang et al. 2012).

New rapid, inexpensive, reliable and reproducible colourimetric methods have been recently developed. In particular, the nitrate reductase assay and resazurin microplate method have been commonly used (Angeby et al. 2002, Coban et al. 2004, Martin et al. 2007, 2011, Palomino et al. 2007, WHO 2010). Farnia et al. (2008)re-

doi: 10.1590/0074-0276130697

+ Corresponding author: yilmazden@hotmail.com

Received 12 December 2012

Accepted 9 September 2013 ported that the colourimetric malachite green decolourisation assay (MGDA) could be used for the rapid detection of resistance.

The aim of this study was to investigate the performance of the MGDA test for the detection of isoniazid (INH) and rifampicin (RIF) resistance in M. tuberculosis clinical isolates.

\section{MATERIALS AND METHODS}

Bacterial isolates - Fifty M. tuberculosis isolates were tested in this study. Nineteen isolates were multidrug-resistant (MDR), eight isolates were only resistant to INH and the remaining isolates were sensitive to both INH and RIF. The BACTEC 460TB system was used as the reference method for testing of the MDR isolates and the BACTEC MGIT 960 system was used as the reference method for testing the remaining isolates. The concentrations of INH and RIF for the BACTEC $460 \mathrm{~TB} /$ BACTEC MGIT 960 systems were 0.1/0.1 and 2.0/1.0 $\mu \mathrm{g} / \mathrm{mL}$, respectively.

The H37Rv-ATCC 25618 (susceptible to all drugs), ATCC 35822 (resistant to INH), ATCC 35838 (resistant to RIF) and ATCC 35820 (resistant to STR) strains were used for quality control. Ethical approval was not required for this study.

Preparation of antibiotics and malachite green $(M G)$ - The INH and RIF antibiotics used in this study were purchased from Sigma and the MG dye was from Merck (Germany). INH and MG were dissolved in sterile distilled water and RIF was prepared in methanol. Stock solutions were prepared as $1,000 \mu \mathrm{g} / \mathrm{mL}, 1,000 \mu \mathrm{g} / \mathrm{mL}$ and $50 \mu \mathrm{g} / \mathrm{mL}$ for INH, RIF and MG, respectively. All reagents were stored at $-20^{\circ} \mathrm{C}$ until use. 
Preparation of bacterial inocula - Bacterial colonies were freshly grown on Löwenstein-Jensen media, transferred into tubes containing physiologic saline and 15-20 glass beads and vortexed for $30 \mathrm{sec}$. The tubes were kept in a vertical position for $30 \mathrm{~min}$ at room temperature to allow for the sedimentation of aerosols and large particles. The turbidity of the supernatant was adjusted to that of the McFarland 1 standard.

Preparation of media - All M. tuberculosis isolates were tested in Middlebrook 7H9S broth (containing $0.1 \%$ casitone, $0.5 \%$ glycerol and $10 \%$ OADC). INH and RIF were tested at the critical concentrations of $0.25 \mu \mathrm{g} /$ $\mathrm{mL}$ and $0.5 \mu \mathrm{g} / \mathrm{mL}$, respectively (Martin et al. 2011). An antibiotic-free growth control tube was also used for each bacterium.

Performing the MGDA test - Three tubes containing INH, RIF or antibiotic-free control growth medium were used for each bacterium. Fifty microlitres of inoculum adjusted to the McFarland 1 standard was added to each tube and the tubes were incubated at $37^{\circ} \mathrm{C}$. Following a seven-day incubation period, $150 \mu \mathrm{L}$ of MG (50 $\mu \mathrm{g} / \mathrm{mL}$ stock solution) was added into each test tube and the tubes were incubated for an additional 24-48 h. Once the MG decolourised in the growth control tube, the test tubes containing antibiotic were evaluated for colour change. If the MG decolourised as a result of bacterial growth in the tubes containing antibiotic, the bacterial sample was reported as resistant (Figure).

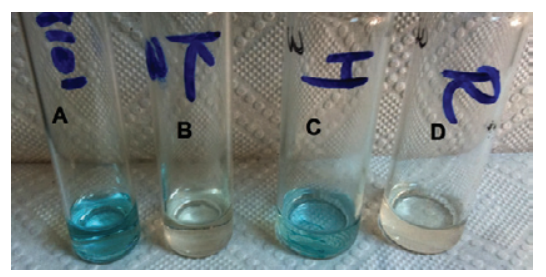

A: negative control (not contain drug and bacteria); B: positive growth control; C: isoniazid-susceptible (there is no growth, colour was not decolourised); D: rifampicin-resistant (decolourisation was observed due to bacterial growth).

\section{RESULTS}

The sensitivity, specificity, positive predictive value (PPV), negative predictive value (NPV) and agreement of the MGDA test for INH were $92.5 \%, 91.3 \%, 92.5 \%$, $91.3 \%$ and $92 \%$, respectively. Two isolates were sensitive to INH by the MGDA test, but resistant by the reference method. Two additional isolates were sensitive to INH by the reference method, but resistant by the MGDA test. For RIF, the sensitivity, specificity, PPV, NPV and agreement of the MGDA test were $94.7 \%, 100 \%, 100 \%, 96.8 \%$ and $98 \%$, respectively. One isolate was resistant to RIF by the reference method, but susceptible by the MGDA test (Table). The results were obtained within eight-nine days.

\section{DISCUSSION}

Phenotypic methods that are inexpensive, rapid and reliable make susceptibility testing of $M$. tuberculosis easier and more efficient. Studies have shown that colourimetric tests are promising methods for drug susceptibility testing, particularly the resazurin microplate method, the resazurin tube method, the nitrate reductase test, the MTT and the XTT (Angeby et al. 2002, de Logu et al. 2003, Montoro et al. 2005, Coban et al. 2006, Bwanga et al. 2010, Dixit et al. 2012).

MG is a triphenylmethane dye that has antimicrobial effects. MG has been widely used to prevent fungal infections in fish. In contrast to most bacteria and fungi, mycobacteria are resistant to MG. Media for the cultivation of mycobacteria (Löwenstein-Jensen) contain MG to reduce overgrowth by contaminating microorganisms. MG can be reductively decolourised. Mycobacterial resistance to MG may be due to dye reduction and sequestration in the lipid fraction of the cells (Jones \& Falkinham 2003).

Gelman et al. (2012) reported that MG interfered with the recovery of mycobacteria on solid culture media following exposure to certain antibiotics, including INH and ethionamide (ETM), that target cell wall biogenesis. This interference did not affect the test results for INH sensitivity because MG was used to determine the viability of bacteria in this test.

Farnia et al. (2008) reported that MGDA could be used for the rapid detection of drug susceptibilities of $M$.

TABLE

Comparing the results of malachite green decolourisation assay (MGDA) with those obtained with reference method

\begin{tabular}{lcccccccc}
\hline & \multicolumn{9}{c}{$\begin{array}{c}\text { Reference method } \\
(\mathrm{n})\end{array}$} & \multicolumn{2}{c}{$\begin{array}{c}\text { Sensitivity } \\
\text { Drug }\end{array}$} & MGDA & $\mathrm{R}$ & $\mathrm{S}$ & $\begin{array}{c}\text { Specificity } \\
(\%)\end{array}$ & $\begin{array}{c}\text { PPV } \\
(\%)\end{array}$ & $\begin{array}{c}\text { NPV } \\
(\%)\end{array}$ & $\begin{array}{c}\text { Agreement } \\
(\%)\end{array}$ \\
\cline { 2 - 9 } INH & $\mathrm{R}$ & 25 & 2 & 92.5 & 91.3 & 92.5 & 91.3 & 92 \\
& $\mathrm{~S}$ & 2 & 21 & & & & & \\
\multirow{2}{*}{ RIF } & $\mathrm{R}$ & 18 & 0 & 94.7 & 100 & 100 & 96.8 & 98 \\
& $\mathrm{~S}$ & 1 & 31 & & & & & \\
\hline
\end{tabular}

INH: isoniazid; NPV: negative predictive value; PPV: positive predictive value; R: resistant; RIF: rifampicin; S: susceptible. 
tuberculosis clinical isolates; the sensitivity, specificity and agreement of the MGDA test were found to be $100 \%$ for both RIF and INH and the results were obtained within six-17 days (average of 12 days). In our study, the INH-RIF sensitivities, specificities, PPVs, NPVs and agreements of the MGDA test were $92.5-94.7 \%$, 91.3$100 \%, 92.5-100 \%, 91.3-96.8 \%$ and $92-98 \%$, respectively and the results were obtained within eight-nine days. There was a major discrepancy in the tests of two isolates that were sensitive to INH by the MGDA test, but resistant by the reference method. In addition, there was a minor discrepancy in the tests of two additional isolates that were sensitive to INH by the reference method, but resistant by the MGDA test.

There is an inconsistency in the first study published by Farnia et al. (2008). The group reported that $50 \mu \mathrm{L}$ of a $0.02 \mu \mathrm{g} / \mathrm{mL}$ MG stock solution was used for the direct susceptibility test, whereas $50 \mu \mathrm{L}$ of a $0.02 \mathrm{mg} / \mathrm{mL}$ MG stock solution was used for the indirect susceptibility test. Therefore, the concentration of the stock solution used in the study is not clear. Our preliminary studies indicated that using a $0.02 \mu \mathrm{g} / \mathrm{mL}$ stock solution was not suitable for the MGDA test; an MG titration showed that $150 \mu \mathrm{L}$ of a $150 \mu \mathrm{g} / \mathrm{mL}$ stock solution was suitable for the susceptibility testing using $1 \mathrm{~mL}$ test tubes (Figure). Mirabal et al. (2010) reported that MGDA could be used for the rapid detection of pyrazinamide resistance. They used $50 \mu \mathrm{L}$ of a $50 \mu \mathrm{g} / \mathrm{mL}$ MG solution in their study. No additional studies using MGDA were found in searches of Medline, PubMed, ISI Web, Web of Science and Google Academic databases.

In conclusion, the MGDA test for the rapid detection of INH and RIF resistance is an inexpensive, easy to perform and interpretable method that can be used in laboratories for routine drug susceptibility testing.

\section{REFERENCES}

Angeby KAK, Klintz L, Hoffner SE 2002. Rapid and inexpensive drug susceptibility testing of Mycobacterium tuberculosis with a nitrate reductase assay. J Clin Microbiol 40: 553-555.

Bwanga F, Hoffner S, Haile M, Joloba ML 2009. Direct susceptibility testing for multidrug resistant tuberculosis: a meta-analysis. BMC Infect Dis 9: 67.

Bwanga F, Joloba ML, Haile M, Hoffner S 2010. Evaluation of seven tests for the rapid detection of multidrug-resistant tuberculosis in Uganda. Int J Tuberc Lung Dis 14: 890-895.

Chang K, Lu W, Wang J, Zhang K, Jia S, Li F, Deng S, Chen M 2012. Rapid and effective diagnosis of tuberculosis and rifampicin resistance with Xpert MTB/RIF assay: a meta-analysis. $J$ Infect 64 : 580-588.

Coban AY, Birinci A, Ekinci B, Durupinar B 2004. Drug susceptibility testing of Mycobacterium tuberculosis with nitrate reductase assay. Int J Antimicrob Agents 24: 106-108.

Coban AY, Cihan CC, Bilgin K, Uzun M, Akgunes A, Cetinkaya E, Durupinar B 2006. Rapid susceptibility test for Mycobacterium tuberculosis to isoniazid and rifampin with resazurin method in screw-cap tubes. $J$ Chemother 18: 140-143.

de Logu A, Pellerano ML, Sanna A, Pusceddu MC, Uda P, Saddi B 2003. Comparison of the susceptibility testing of clinical isolates of Mycobacterium tuberculosis by the XTT colorimetric method and the NCCLS standards method. Int J Antimicrob Agents 21: 244-250.

Dixit P, Singh U, Sharma P, Jain A 2012. Evaluation of nitrate reduction assay, resazurin microtiter assay and microscopic observation drug susceptibility assay for first line antitubercular drug susceptibility testing of clinical isolates of M. tuberculosis. J Microbiol Methods 88: 122-126.

Farnia P, Masjedi MR, Mohammadi F, Tabarsei P, Farnia P, Mohammadzadeh AR, Baghei P, Varahram M, Hoffner S, Velayati AA 2008. Colorimetric detection of multidrug-resistant or extensively drug-resistant tuberculosis by use of malachite green indicator dye. J Clin Microbiol 46: 796-799.

Friedrich SO, Venter A, Kayigire XA, Dawson R, Donald PR, Diacon AH 2011. Suitability of Xpert MTB/RIF and Genotype MTBDRplus for patient selection for a tuberculosis clinical trial. J Clin Microbiol 49: 2827-2831.

Gelman E, McKinney JD, Dhar N 2012. Malachite green interferes with postantibiotic recovery of mycobacteria. Antimicrob Agents Chemother 56: 3610-3614.

Jones JJ, Falkinham III JO 2003. Decolorization of malachite green and crystal violet by waterborne pathogenic mycobacteria. Antimicrob Agents Chemother 47: 2323-2326.

Kent PT, Kubica GP 1985. Public health mycobacteriology: a guide for the level III laboratory, US Department of Health and Human Services/Public Health Service/Center for Disease Control and Prevention, Atlanta, $207 \mathrm{pp}$.

Martin A, Paasch F, Docx S, Fissette K, Imperiale B, Ribón W, González LA, Werngren J, Engström A, Skenders G, Juréen P, Hoffner S, del Portillo P, Morcillo N, Palomino JC 2011. Multicentre laboratory validation of the colorimetric redox indicator (CRI) assay for the rapid detection of extensively drug-resistant (XDR) Mycobacterium tuberculosis. J Antimicrob Chemother 66: 827-833.

Martin A, Panaiotov S, Portaels F, Hoffner S, Palomino JC, Angeby $\mathrm{K} 2008$. The nitrate reductase assay for the rapid detection of isoniazid and rifampicin resistance in Mycobacterium tuberculosis: a systematic review and meta-analysis. $J$ Antimicrob Chemother 62: 56-64.

Martin A, Portaels F, Palomino JC 2007. Colorimetric redox-indicator methods for the rapid detection of multidrug resistance in Mycobacterium tuberculosis: a systematic review and meta-analysis. J Antimicrob Chemother 59: 175-183.

Mirabal NC, Yzquierdo SL, Lemus D, Madruga M, Milián Y, Echemendía M, Takiff H, Martin A, van der Stuyf P, Palomino JC, Montoro E 2010. Evaluation of colorimetric methods using nicotinamide for rapid detection of pyrazinamide resistance in Mycobacterium tuberculosis. J Clin Microbiol 48: 2729-2733.

Montoro E, Lemus D, Echemendia M, Martin A, Portaels F, Palomino JC 2005. Comparative evaluation of the nitrate reductase assay, the MTT test and the resazurin microtiter assay for drug susceptibility testing of clinical isolates of Mycobacterium tuberculosis. $J$ Antimicrob Chemother 55: 500-505.

Palomino JC, Martin A, Portaels F 2007. Rapid drug resistance detection in Mycobacterium tuberculosis: a review of colourimetric methods. Clin Microbiol Infect 13: 754-762.

WHO - World Health Organization 2010. Non-commercial culture and drug-susceptibility testing methods for screening of patients at risk of multi-drug resistant tuberculosis: policy statement. Available from: who.int/tb/publications $/ 2011 / \mathrm{mdr}_{-}$tb diagnostics_9789241501620/en/. 